\title{
Lipoma de la cisterna cuadrigémina y cisura calcarina: caso clínico y revisión de la literatura
}

\author{
J. Fandiño; J. Bermúdez* y E. Arán
}

Unidad de Neurocirugía. *Servicio de radiología. Hospital Xeral Calde Lugo.

\section{Resumen}

Presentamos el caso de un varón de 47 años, portador de dos lipomas intracraneales, localizados en la lámina cuadrigémina y cisura calcarina respectivamente, lo que consideramos un caso excepcional.

El paciente padecía cefaleas, episodios vertiginosos y cuadrantanopsia. Se describen los hallazgos en la Resonancia Magnética (RM) y realizamos una breve revisión de la literatura.

PALABRAS CLAVE. Lipoma intracraneal múltiple. Cisura calcarina. Cisterna cuadrigémina.

Quadrigeminal cistern and calcarine fissure lipoma: case report and review of the literature

\section{Summary}

We report the case of a multiple intracranial lipoma in a 47 year-old patient presenting with headache dizziness and cuadrantanopsia. MR imaging revealed the presente of 2 lipomas, one located within the cuadrigeminal cistern and the other in the calcarine fissure. To our knowledge, this combination of this rare lesion has not been yet described. We review the current literature.

KEY WORDS: Multiple Intracranial lipoma. Calcarina fissure. Cuadrigeminal cistern.

\section{Introducción}

Los lipomas intracraneales son lesiones raras, resultantes de la persistencia anormal de la meninge primitiva, como fue descrito por $\operatorname{Verga}^{37} \mathrm{y}$ aceptado por Truwit $\mathrm{y}$ Barkovich $^{35}$. Constituyen el 0,1\% de las lesiones de esta localización ${ }^{10}$, teniendo lugar la mayoría en la línea media $\mathrm{y}$ principalmente en la cisterna pericallosa ${ }^{23}$. La primera mención de la existencia de un lipoma intracraneal es recogida por Meckel, quien en 1818 descubre un lipoma quiasmático durante una autopsia, pero es Rokitansky el primero en describirlo en 1856 asociado a una agenesia del cuerpo calloso ${ }^{11,30,34}$. Se estima su incidencia entre el 0,08\% y el $0,2 \%$ de los casos de autopsia y de un 0.06 a un 0.3 entre los diagnosticados radiológicamente de forma accidental $^{3}$. La mitad de las lesiones se asocian a malformaciones de diverso grado ${ }^{11}$. La existencia de lipomas múltiples intracraneales, si bien ha sido ya descrita ${ }^{25,33}$, es excepcional. La RM es el método diagnóstico de elección con señal hiperintensa en $\mathrm{T} 1^{5,13,35}$. El tratamiento conservador es la regla tanto en lipomas únicos como múltiples ${ }^{11,16,32}$. Presentamos un paciente de 47 años con lipoma múltiple localizado a nivel de cisura calcarina y cisterna cuadrigémina, que fue seguido durante 2 años, sin tratamiento quirúrgico.

Si ya los lipomas múltiples son excepcionales, en la literatura consultada no hemos hallado un caso similar. Se revisa la misma haciendo hincapié en la histogénesis, diagnóstico y tratamiento.

\section{Caso clínico}

Se trata de un varón de 47 años de edad, sin antecedentes de interés, con historia de cefalea y ocasionalmente vértigo en los últimos cuatro años, que consulta por trastorno inespecífico de la visión. La exploración neurológica resultó ser normal, excepto la detección de una cuadrantanopsia homónima inferior derecha en el estudio campimétrico. No existían malformaciones o anomalías asociadas a nivel de sistema nervioso central ni fuera de éste. La tomografía computarizada (TC) resultó ser normal. Se le practicó un estudio con RM que demostró, en secuencias T1 y en proyección parasagital (Fig 1, A y B), dos focos de hiperseñal situados en la cisterna cuadrigémina y en el labio superior de la cisura calcarina izquierda compatibles con el diagnóstico de lipomas .

No se realizó intervención quirúrgica, permaneciendo el paciente con idéntica sintomatología dos años después. 


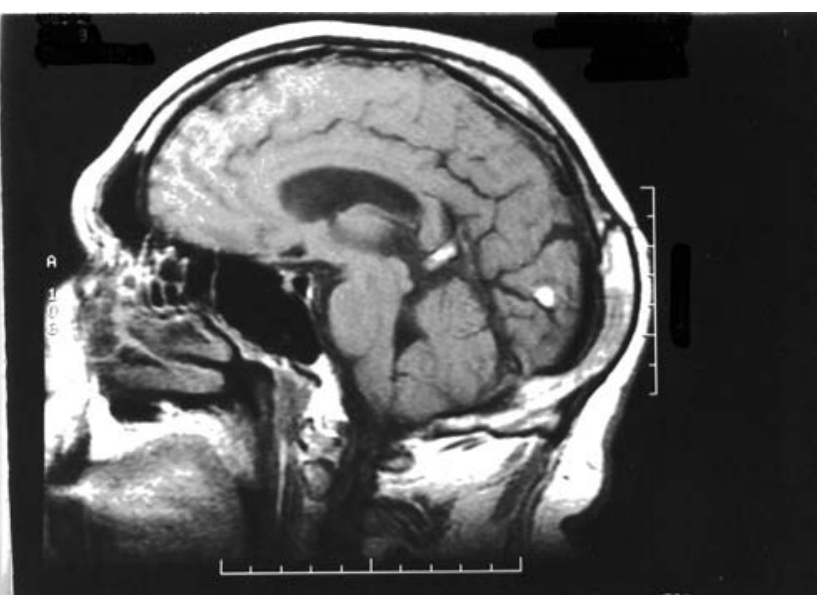

Figura 1 A. Imagen parasagital de RM; secuencias potenciadas en T1 visualizan dos focos de hiperseñal a nivel de cisterna cuadrigémina y cisura calcarina, con características de grasa y diagnóstico de lipoma.

\section{Discusión}

\section{Incidencia}

Los lipomas intracraneales se consideran como lesiones raras dentro del sistema nervioso. La existencia de lipomas múltiples debe considerarse, por lo tanto, como algo excepcional, como así lo demuestra la escasa literatura encontrada sobre el tema. Entre los casos más significativos citaremos el reportado por Hädecke ${ }^{17}$ con sendos lipomas localizados en la cisterna opto-quiasmática y lóbulo temporal. Wilson ${ }^{38}$ hace mención a la coexistencia de un lipoma originado en la médula cervical superior, con extensión a la fosa posterior, asociado a dos lipomas de la región lumbar. Por su parte Cohen ${ }^{8}$ describe la existencia de múltiples lipomas en los ventrículos cerebrales y cisternas basales, fruto de "émbolos" de un lipoma del cono medular. No es infrecuente la asociación de lipomas del cuerpo calloso y lipomas del plexo coroideo, no siempre con una solución de continuidad a través de la fisura coroidal como cita Melin ${ }^{26}$ y describe $S^{2} a^{33}$ en un paciente con un gran lipoma pericalloso y múltiples lipomas del plexo coroideo. Sí han sido descritos lipomas múltiples intracraneales formando parte del síndrome de Goldenhar-Gorlin ${ }^{1,11,12}$ o del síndrome de Bannayan, en donde cohabitan con otras malformaciones del sistema nervioso, incluidos tumores cerebrales, pero queda claro que nuestro caso no forma parte de estos síndromes.

\section{Definición y Origen}

El término lipoma conduce a pensar que nos encontrarnos ante una neoplasia, pero en realidad se trata de un

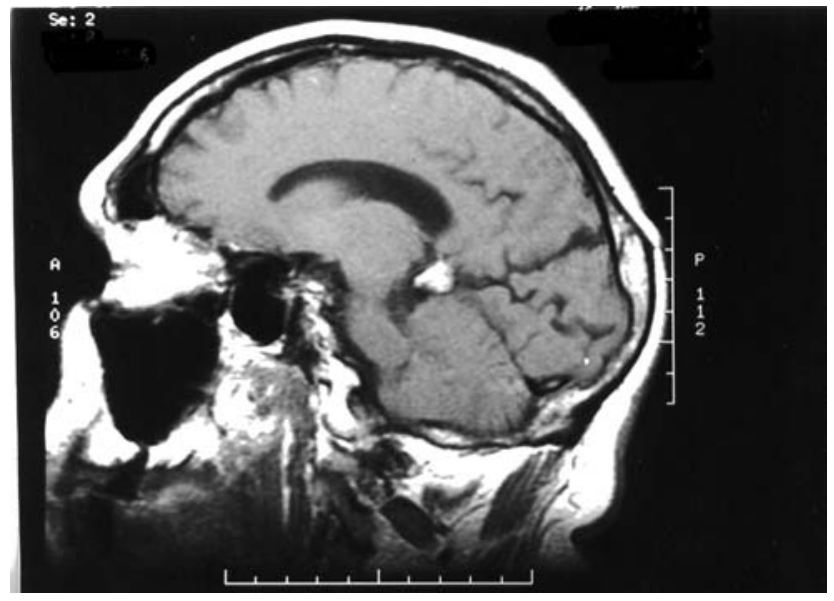

Figura 1 B. En otro corte parasagital de RM sólo se detecta el lipoma ocupante de la cisterna cuadrigémina pero en su máximo volumen.

defecto embriológico. En 1929, Verga ${ }^{37}$ relaciona el origen de los lipomas intracraneales con la meninge primitiva; es Krainer $^{23}$ quien recoge esa teoría señalando la naturaleza cisternal y el origen no neoplásico de los lipomas, llegando a afirmar que un foco persistente de meninge primitiva induciría a la diferenciación del tejido adiposo y a la aparición del lipoma. Es sabido que el plexo coroideo del IV ventriculo no se forma hasta el estadio 19 de Carnegie, hacia el día 48, y la cavitación meníngea tiene lugar entre el día 32 y 44, las cisternas perimensencefálicas y el mesencéfalo dorsal se forman sobre el día 41 de gestación; así pues, el espacio subaracnoideo se ha formado antes que el plexo coroideo del IV ventriculo. Sobre el estadio 20 de Carnegie la mayor parte de la meninge es reabsorbida, quedando concentrada sobre la cara dorsal de la médula y la lamina terminalis. A los 56 días de gestación (estadio 23) sólo persiste una mínima colección de meninge dorsorostral a la lamina terminalis y, debido a este "retraso" en la desaparición, es esta zona donde la posibilidad de una mala diferenciación de la meninge es mayor y el lugar de mayor frecuencia de aparición de lipomas; una porción de la lamina terminalis es origen de las fibras del cuerpo calloso. La persistencia de la meninge afectaría al cierre del surco mediano y a la formación de las masas comisurales, lo que se traduciría en una hipoplasia o agenesia del cuerpo calloso. Se podría argumentar que no existe tejido graso en el sistema nervioso, pero sí es aceptado que la meninge primitiva y el parénquima precapilar pueden diferenciarse en grasa $^{3}$.

En conclusión, un lipoma es un espacio subaracnoideo mal diferenciado, con una localización característica dentro del cerebro, que se asocia a malformaciones cerebrales en más del cincuenta por cien de los casos y se confirma que estamos ante una malformación congénita. 


\section{Localización y Diagnóstico}

Es bien conocido que la mitad de los lipomas intracraneales son pericallosos, siendo los siguientes en frecuencia los que asientan en la cisterna ambiens y cuadrigémina que constituyen entre el 20 y $25 \%{ }^{19,20,21,24,27,28}$. Le siguen los que ocupan el ángulo pontocerebeloso con un $9 \%^{31,33}$. En raras ocasiones se localizan en la superficie del hemisferio cerebral ${ }^{14,15}$ y cuando se producen suelen asociarse a malformaciones del sistema nervioso central, siendo la localización en la cisura de Silvio la más habitual, con aproximadamente un 5\% de los lipomas intracraneales. Por tanto, la posibilidad de la localización en otra cisura, en este caso concreto la calcarina, es muy remota y la asociación de las dos localizaciones presentadas la consideramos excepcional, habida cuenta que no hemos encontrado en la literatura ninguna descripción de la asociación de lipoma en la cisura calcarina y cisterna cuadrigémina En general las manifestaciones clínicas incluyen cefalea (25\%), epilepsia $(30 \%)$, trastornos mentales $(15 \%)$, siendo un tercio asintomáticos y la exploración neurológica normal. Efectivamente, el lipoma cuadrigémino no tenía expresión clínica, siendo los de esta localización propensos a provocar ataxia, hidrocefalia, parálisis de la mirada o envolver el nervio troclear ${ }^{2,18}$.

Los lipomas localizados en las cisuras suelen producir epilepsia por afectación del cortex ${ }^{9,14}$ o bien compresión de vasos o nervios que discurren por la zona cisural o cisternal, en el caso del lipoma de la cisura calcarina no se da ninguna de las dos circunstancias y sí una afectación del campo visual propio del área de asiento del lipoma.

La RM es el método de elección para el diagnóstico de los lipomas intracraneales ${ }^{4}$. Aparecen como lesiones hiperintensas en T1 e hipo-isointensas en T2, no existiendo captación de contraste salvo en algunos casos donde el componente vascular del lipoma es importante. El gran valor diagnóstico de la RM ha quedado de manifiesto en este paciente, al serle detectado sendos lipomas en una localización que difícilmente pudiera sospecharse con otros métodos radiológicos (radiología simple o tomografía computarizada) y cuya sintomatología de vértigo, cefalea y trastornos visuales eran escasamente específicos y topográficos a priori. Por otra parte, la localización de ambos lipomas en estructuras de línea media sigue la "ley" de asentamiento intracraneal de la mayoría de lipomas. En lipomas de escaso volumen, como es nuestro caso, el uso de la tomografía computarizada (TC) puede proporcionar falsos negativos ya que si los lipomas son menores que la distancia entre dos cortes de TC pueden pasar desapercibidos o confundirse con efecto de volumen parcial. No así con la RM. en donde el diagnóstico es prácticamente de certeza $^{6,33,36}$. Conviene aclarar, a la vista de las figuras $1 \mathrm{~A}$ y $1 \mathrm{~B}$, que también en la RM pueden aparecer lipomas, es el caso que nos ocupa, que no aparecen en un determinado corte y sí en el siguiente.

\section{Tratamiento}

La actividad proliferativa de estos procesos es muy baja $^{29}$, por lo que el curso biológico es favorable, incluso sin tratamiento quirúrgico. Recordemos que los lipomas no crecen, aunque sí se hipertrofian con el crecimiento somático $^{33}$ si bien, y como rareza, mencionamos el caso descrito por $\mathrm{Haga}^{18}$ en el que un paciente con diagnóstico de arteritis de células gigantes fue tratado con esteroides y desarrolló clínica de visión borrosa, diplopia, parálisis oculomotora izquierda y ptosis palpebral, se realizaron estudios neurorradiológicos cerebrales, diagnosticando un lipoma del cavum de Meckel, lo que llevó a concluir que los lipomas intracraneales pueden aumentar de volumen con el tratamiento esteroideo. Es generalmente aceptado que su extirpación quirúrgica está contraindicada, pero no siempre fue así ya que Clarici y Heppner ${ }^{7}$ extirpan en su totalidad un lipoma calloso utilizando láser de $\mathrm{CO}_{2}$ y Groff et $a{ }^{16}$ dan garantías de éxito en la extirpación de los lipomas del cuerpo calloso siempre y cuando no sea penetrado el III ventrículo. Por error en el diagnóstico preoperatorio. Kazner et $\mathrm{al}^{22}$ intervienen un lipoma de la cisterna cuadrigémina creyendo se trataba de un quiste dermoide.

En el caso que nos ocupa no se realizó cirugía, en vista de las escasas dimensiones de los lipomas y de la sintomatología perfectamente asumida por el paciente.

\section{Bibliografía}

1. Aleksic, S., Budzilovich, G., Greco, M.A., McCartlry, J., Reuben, R., Margolis, S.: Intracranial lipoma, hydrocephalus and other CNS anomalies in oculoauriculo-vertebral dysplasia (Goldenhar-Gorlin syndrome). Childs Brain 1984; 11: 285-297.

2. Anmbrosetto,P., Martinelli, P., Bacci, A., Daidone, R.: Lipoma of the quadrigeminal plate cistern. Ital J Neurol Sci 1985: 6: 347-349.

3. Bailey, F., Bucy, P.C.: The origen and natura of meningeal tumors. Am J Cancer 1931, 15: 15-54.

4. Bakshi, F., Shaikh, Z.A., Kainram, S., Kinkel, P.R.: MRI findings in 32 consecutive lipornas using conventional and advanced sequences. J Neuroimag 1999; 9: 134-140.

5. Barkovich, A.J., Maroldo, T.V.: Magnetic resonance imaging of normal and abnormal brain development. Top Magn Reson Imaging 1993; 5: 96-122.

6. Burger, P.C., Scheithauer, B.W.: Lipoma. En: Rosai, J., Sobin LS (eds). Atlas of tumor patliology. Tumors of the central nervous system. Washington DC, Armed Forces Institute of Patliology, 1994 pp 299-301.

7. Clarici, G., Heppner, F.: The operative approach to lipo- 
mas of the corpus callosum. Neurochirurgia (Stuttg) 1979, 22: 77-81.

8. Cohen, A.R., Murali, R.: Intracranial lipoma ascending to the brain. Surg Neurol 1991, 11: 215-219.

9. Cserni, G.: Intracranial lipoma of the Sylvian region. Neuropathol Appl Neurobiol 1993; 19: 282-285.

10. Donati, F., Vassella, F., Kaiser, G., Blumberg, A.: Intracranial lipomas. Neuropediatrics 1992: 23: 32-38.

11. Fandiño, J.: Lipomas intracraneales. Res, Neurol 2001. 32: 644-650.

12. Friede, R.L.: Osteolipomas of the tuber cinereum. Arch Pathol Lab Med 1977; 101-369-372.

13. Frund, F., Geissler, A., Gliese, M., Seitz, J., Feuerbach, S.: Magnetic resonance imaging (MRI) and magnetic resonance spectroscopy (MRS) of intracranial lipomas. Front Biosci 1997; 2: 13-16.

14. Fujii, Y., Konishi, Y., Kriyama. M., Hori, C., Sudo, M.: Lipoma on surface of centroparietal lobes. Pediatr Neurol 1993; 9: 144-146.

15. Gabrovski, S., Poptodorov, G., Aleksiev, A., Kruster, E., Dimitrov, D.: Lipoma of the temporal lobe. Khirurgiia (Soffia) 1999-155: 55-59.

16. Groff, R.A., Liu, C.T., Leopold, R.L.: Lipoma of the corpus callosum: survey of the literature ans report of the two surgical cases. Arch Neurol Psychiatry 1951; 65: 253-254.

17. Hädecke, J., Buclüelder, M., Triebel, H.J., Schneyer, U.: Multiple intracranial lipoma: a case report. Neurosurg Rev 1997; 20: 282-287.

18. Haga, H.J., Thomassen, E., Johannesen, A., Krakenes, J.: Neural compressive symptoms appearing during steroid treatment in a patient with intracranial lipoma. Scand J Rheumatol 1999; 28-184-186.

19. Halmagyi, G.M., Evans, W.A.: Lipoma of the quadrigeminal plate causing progressive hydrocephalus. Case report. J neursurg 1978; 49: 453-456.

20. Hori, A.: Lipoma of the quadrigeminal region with evidence of congenital origen. Arch Pathol Lab Med 1986: 110: 850-851.

21. Kawamata, T., Aoki, N., Sakai, T., Takakura, K.: Congenita triventricular hydrocephalus associaled with a small lipoma in the quadrigeminal plate cistern. Childs Nerv Sysi 1995; 11: 121-123.

22. Kazner, E., Stochdorph, O., Wende, S., Grumme, T.: Intracranial lipoma: diagnostic and therapeutic considerations. J Neurosurg 1980; 52: 234-245.

23. Krainer, L.: Die Hirn-und Rückenmarkslipome. Virchows Arch (Pathol Anat) 1935-,295: 107-142.

24. Maiuri, F., Corriero, G., Gallichio, B., Simoneti, L.: Lipoma of the ambient cistern causing hydrocePhalus. J Neurosurg Sci 1987; 31: 51-58.
25. Meitner, E.R.: Lipoma duplex regionis callosi. Psychiatr Neurol Med Psychol 1962; 14: 384-385.

26. Melin, G.I., Keller, M.S.: pericallosal lipoma axtending through the coroidal Gssure: US/CT/MRT Correlation. Neuroradiology 1992; 34: 402-403.

27. Nikaido, Y., Imanishi, K., Monobe, T.: Lipoma in the quadrigeminal cistern. Case report. Neurol Med Chir (Tokyo) 1995; 35: 175-178.

28. Ono, J., Ikeda, T., Imai, K., Matsuoka, T., Nagai, T., Okada, S.: Iniracranial lipoma of the quadrigeminal region associated with complex partial seizures. Pediatr Radiol 1998; 28: 729-731.

29. Pilcher, W.H., Locharernkul, C., Primrose, D., Moretti Ojemann, L., Ojemann, G.A.: Update in epilepsy; Part III: Surgical Therapy of intractable epilepsy. New York State J Med 1992; 92: 92-96.

30. Rokitansky, C.: Lehrbuch der Pathologischen anatomie. Vol 2. Viena: Braumuller; 1856. P. 33.

31. Román, A.M., Olivares, G., Katati, M., Horcajadas, A., Arjona, V.: Lipoma del ángulo pontocerebeloso: caso clínico. Neurocirugía 2002; 1: 38-45.

32. Saatci, I., Aslan, C., Renda, Y., Besim, A.: Parietal lipoma associated with cortical dysplasia and abnormal vasculature: Case report and review of literature. AJNR 2000, 21 : 1718-1721.

33. Shah, J., Srinivase, P., Gale, B., Patkar, D., Patankar, T., Kale, H.: Multiple intracranial lipomas, hypogenetic corpus callosum and vestibular schwannoma: an unusual spectrum of RM findings in a Patient. J Postgrad Med 1999; 45: 53-55.

34. Suzuki, M., Takashima, T., Kadoya, M., Ueda, F., Arai, K., Arakawa, F., et al: Pericallosal lipomas: MR features. J Comput Assist Tomogr 1991; 15: 207-209.

35. Trwit, C.L., Barkovich, A.J.: Patogenesis of intracranial lipoma: an MR siudy in 42 patients. Am J Neuroradiol 1990; 11:665-674.

36- Uchino, A., Hasuo K.: Solitary choroid plexus lipomas: CT and MR appearance. Am J Neurradiol 1993; 14: 116118.

37. Verga, P.: Lipoma ed osteolipomi della pía madre. Tumori 1929; 15: 321-357.

38. Wilson, J.T., Shapiro, R.H., Wald, S.L.: Multiple intradural spinal lipomata with intracranial extension. Pediatr Neurosurg 1996; 24: 5-7.

Fandiño, J.; Bermúdez, J.; Aran, E.: Lipoma de la cisterna cuadrigémina y cisura cálcarina: caso clínico y revisión de la literatura. Neurocirugía 2005; 16: 173-176.

Correspondencia postal: J. Fandiño. C/Montevideo 17- 3. 27001 Lugo. España. 